\title{
Right visual-field advantage in the attentional blink: Asymmetry in attentional gating across time and space
}

\author{
Dafna Bergerbest $^{1} \cdot$ Orit Shilkrot $^{1} \cdot$ Michal Joseph $^{1} \cdot$ Moti Salti $^{2,3}$
}

Published online: 22 June 2017

(C) The Psychonomic Society, Inc. 2017

\begin{abstract}
When two targets are presented in a rapid serial visual presentation (RSVP), recognition of the second target (T2) is usually reduced when presented 150-500 ms after the first target, demonstrating an attentional blink (AB). Previous studies have shown a left visual-field (LVF) advantage in T2 recognition, when $\mathrm{T} 2$ was embedded in one of two streams, demanding top-down attention for its recognition. Here, we explored the impact of bottom-up saliency on spatial asymmetry in the AB. When T2 was spatially shifted outside from the RSVP, creating an abrupt onset of T2, right T2s showed a right visual-field (RVF) advantage. In lag-1 trials, right T2s were not only better recognized, but also showed a low T1-T2 order error rate. In contrast, recognized left $\mathrm{T} 2 \mathrm{~s}$ exhibited high order error rate. Without abrupt onset, symmetrical AB was found and order error rate was similarly low in both sides. Follow-up experiments showed that, while RVF advantage was related to bottom-up saliency, order errors were affected by T1 mask. The discrepancy between LVF and RVF advantage in the AB could be resolved in terms of two mechanisms of attentional gating: top-down attentional gating, which is biased towards LVF, and bottom-up attentional gating, which is biased towards RVF.
\end{abstract}

Dafna Bergerbest

dafnaberg@gmail.com

1 School of Behavioral Sciences, The Academic College of Tel-Aviv-Yaffo, Tel Aviv-Yafo 6818211, Israel

2 Brain Imaging Research Center, Ben-Gurion University of the Negev, Beer-Sheva 8410501, Israel

3 Zlotowski Center for Neuroscience, Ben-Gurion University of the Negev, Beer-Sheva 8410501, Israel
Keywords Attentional blink · Visual awareness · Temporal processing

The attentional blink $(\mathrm{AB})$ is a deficit in reporting the second of two targets presented in close temporal succession (Raymond, Shapiro, \& Arnell, 1992). The AB has been a central phenomenon in attention and consciousness research for more than two decades as it reveals the temporal dynamics of stimuli encoding and its access to consciousness (for reviews, see Dux \& Marois, 2009; Martens \& Wyble, 2010).

The AB is typically studied using a rapid serial visual presentation (RSVP), where two visually presented targets (e.g., digits) are embedded in an RSVP (e.g., letters), and participants are asked to report the two targets. The second target (T2) is often missed if it follows, or lags, the first target (T1) within an interval of about 150-500 ms (Broadbent \& Broadbent, 1987; Raymond et al., 1992).

Theoretical accounts of the AB have assumed limited attentional resources that are exhausted on T1 processing in the expense of T2 processing (e.g., Chun \& Potter, 1995; Jolicoeur \& Dell'Acqua, 1998; Shapiro, Raymond, \& Arnell, 1994). All these accounts predict that the AB deficit would be most pronounced when T2 directly trails T1, in lag 1. Contrary to this prediction, a meta-analysis by Visser, Bischof, and Di Lollo (1999) revealed that, in approximately half of the studies, T2s presented in lag 1 escaped the blink, yielding a U-shaped AB function. Potter, Chun, Banks, and Muckenhoupt (1998) referred to this highly accurate performance at lag 1 as Lag-1 sparing.

Superior report accuracy at lag 1 typically comes with a cost of impaired temporal order perception. That is, participants are likely to perceive both $\mathrm{T} 1$ and $\mathrm{T} 2$, but report them, on substantial proportion of the trials (often $25 \%$ or more) in the reverse order (e.g., Akyürek, Toffanin, \& Hommel, 2008; 
Akyürek et al., 2012, Exp. 5; Hommel \& Akyürek, 2005; Olivers, Hilkenmeier, \& Scharlau, 2011; Potter, Staub, \& O'Connor, 2002; Shih, 2000; Wyble, Bowman, \& Nieuwenstein, 2009). Because sparing and order errors that accompany lag 1 performance restrict the theoretical accounts for the $\mathrm{AB}$, the conditions under which they appear are informative for its underlying mechanisms.

Visser and colleagues' (1999) meta-analysis also revealed that Lag-1 sparing disappears when there is a dimensional switch between T1 and T2 (e.g., location, modality, task, and category). Specifically, location shift was the most pronounced dimensional switch that eliminated Lag-1 sparing. Visser, Zuvic, Bischof and Di Lollo (1999) directly demonstrated Lag-1 sparing elimination when T2 was spatially shifted relative to T1, and concluded that "the presentation of a target triggers the opening of an attentional gate that is tied to the spatial location of that target" (p. 436). Visser, Zuvic et al. (1999) added that, if a gate is currently open, it must first be closed before a new gate could be opened elsewhere.

Indeed, most studies that tested the effects of presenting T1 and $\mathrm{T} 2$ in different locations did not find Lag-1 sparing. ${ }^{1}$ These studies used dual-stream RSVP, where the two targets were embedded in two separate visual streams, located to the left and to the right of a fixation point (but see Giesbrecht \& Kingstone, 2004; Scalf, Banich, Kramer, Narechania, \& Simon, 2007; Visser, Zuvic et al., 1999). Interestingly, these studies reported a left visual-field (LVF) advantage, with T2 being better recognized when presented on the left stream, following $\mathrm{T} 1$ presentation in the right stream, than the reverse. This LVF advantage was demonstrated at lag 1 as well as in later lags (e.g., Asanowicz, Smigasiewicz, \& Verleger, 2013; Hollander, Corballis, \& Hamm, 2005; Hollander, Hamm, Hausmann, \& Corballis, 2005; Scalf et al., 2007; Śmigasiewicz et al., 2010; Smigasiewicz \& Verleger, 2014; Verleger et al., 2009, 2010; Verleger, Dittmer, \& Smigasiewicz, 2013; Verleger, Smigasiewicz, \& Moller, 2011; for a review see Verleger \& Smigasiewicz, 2015). Note that in most of these studies there was an additional dimension switch between T1 and T2 (e.g., task or category), reducing the likelihood of finding Lag-1 sparing and making order errors practically impossible.

Verleger and Smigasiewicz (2015) interpreted LVF advantage in processing of $\mathrm{T} 2$ as resulting from $\mathrm{T} 2$ being processed by the right hemisphere, which is suggested by them to be "superior in directing attention reactively to salient events"

\footnotetext{
${ }^{1}$ Several recent studies did demonstrate Lag-1 sparing when T1 and T2 were presented in two different RSVP streams (Jefferies \& Di Lollo, 2009; Jefferies, Enns, \& Di Lollo, 2014; Jefferies, Ghorashi, Kawahara, \& Di Lollo, 2007; Potter, Staub, \& O'Connor, 2002; Shih, 2000). However, Jefferies and colleagues suggested that participants' attention was spread across the two streams, leading the two streams to be processed as one location. Lunau and Olivers (2010) reported a more convincing demonstration of Lag-1 sparing for location-shifted $\mathrm{T} 2 \mathrm{~s}$, in a study where attention was shifted continuously across multiple locations by the aid of a moving cue.
}

(p. 266). Note, however, that in these studies T2 was embedded within a stream of distractors, requiring top-down attention in order to be selected for further processing. In fact, the LVF advantage disappeared when the relative salience of $\mathrm{T} 2$ was increased either by pre-cueing T2 location (Smigasiewicz, Asanowicz, Westphal, \& Verleger, 2014), by coloring both targets (Scalf et al., 2007, Exp. 4; Fig. 8 in Verleger \& Smigasiewicz, 2015), or by decreasing targetsdistractors similarity (Scalf et al., 2007, Exps. 2, 3 and 5, but see Exp. 6).

The aim of the present study was to explore whether an asymmetry in spatial shift effects on the $\mathrm{AB}$ and specifically at lag 1 performance could also be found under bottom-up saliency. In four experiments, the bottom-up saliency of T2 was manipulated by means of abrupt onset (Yantis, 1993). In all four experiments, both targets were from the same category (i.e., digits) and required the same task (i.e., recognition), increasing the likelihood of Lag-1 sparing and allowing measuring T1-T2 order errors. These conditions enabled an exhaustive examination of the $\mathrm{AB}$ asymmetry under various levels of T2 bottom-up saliency.

\section{Experiment 1a}

In Exp. 1a, spatial asymmetry in the $\mathrm{AB}$ under conditions of the high bottom-up saliency of T2 was studied using one RSVP stream. T1 was presented on one central stream and $\mathrm{T} 2$ was either presented on that same central stream or shifted to the right or to left. This resulted in shifted-T2s having an abrupt onset, benefiting from high bottom-up saliency. The distractors that followed $\mathrm{T} 2$ were presented at the same location as T2. In order to exhaust the information concerning the sparing, we also recorded T1-T2 order errors. As we were mainly interested in the asymmetry of lag 1 performance, we compared the sparing and order errors not only between lag 1 and 2 but also between left and right.

\section{Method}

\section{Participants}

Twenty undergraduate students (five males, aged 19-26 years) from the Academic College of Tel-Aviv-Yaffo participated for class credit. All reported normal or corrected-tonormal vision. One participant was excluded from the analysis because of a low $\mathrm{T} 1$ recognition rate, deviating below the group mean by more than 2.5 SDs.

\section{Apparatus}

All stimuli were presented on an Intel core i3 computer by a CRT screen with a refresh rate of $75 \mathrm{~Hz}$. The experiment was 
programed and run with E-Prime Professional 2.0. Viewing distance was about $60 \mathrm{~cm}$.

\section{Stimuli}

The target items were digits (2-9), not including 0 and 1 . The distractors were A-Z letters, not including I, O, Q and S due to their similarity to the numbers 1,0 and 5 . The digits and letters were white, in font type David and font size 24 , extending $\sim 0.5^{\circ}$ in width and $\sim 0.75^{\circ}$ in height, presented on a black background.

\section{Procedure}

Participants initiated each trial by pressing on the spacebar when they were ready. Each trial started with a fixation cross that was presented in the center of the screen for $507 \mathrm{~ms}$ (see Fig. 1). Then, the RSVP stream was presented in the center of the screen, each item displayed for $107 \mathrm{~ms}$. The stream was composed as follows: 3-5 distractors, T1, 0, 1, 3, 5 or 6 distractors, T2 and 2-4 distractors. T2 was randomly presented either at the center of the screen, $\sim 1.5^{\circ}$ to the left of center or $\sim 1.5^{\circ}$ to the right of center. The following distractors were presented at the same location as T2. On each trial, the two targets were selected randomly without replacement, so T1 and T2 always differed. All consecutive distractors in the stream were selected randomly without replacement from the set of letters. At the end of the RSVP, participants were asked to indicate what was the first target and what was the second target by typing them on the keyboard. If the participant did not see one of the targets, she was asked to press the spacebar. The following trial started after a $187 \mathrm{~ms}$ interval. Running 25 trials for each combination of T2 Location (Left, Center, or Right) and T1-T2 Lag (1, 2, 4, 6, 7), in a random order, resulted in a total of 375 trials. A short break was offered every 50 trials. The experimental session was preceded

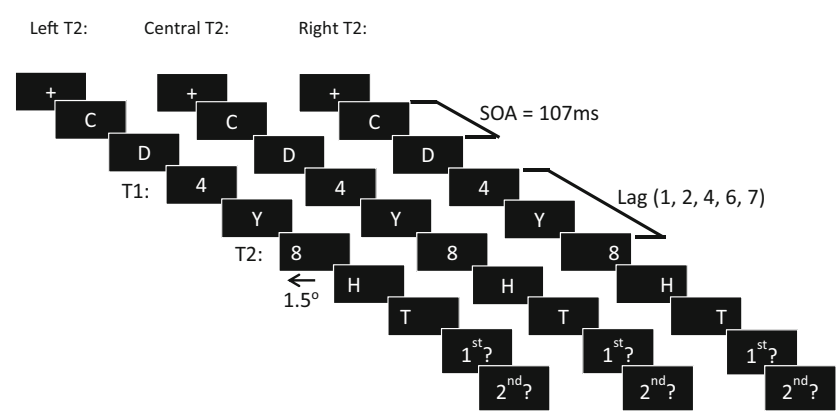

Fig. 1 Experiment 1a. One-stream RSVP with a digit T1 embedded in a stream of letter distractors at the central position. T2 and the following distractors were presented on the center or shifted $1.5^{\circ}$ to the left or to the right. T2 could lag behind T1 in the 1st, 2nd, 4th, 6th or 7th position. Lag 2 is illustrated by a practice session that included 15 trials. The whole session lasted about $40 \mathrm{~min}$.

\section{Results and discussion}

In this and the following experiments, $\mathrm{T} 1$ and $\mathrm{T} 2$ reports were considered as correct regardless of report order. Order errors were analyzed separately.

\section{T1 recognition rate}

A repeated measures $3 \times 5$ analysis of variance (ANOVA) on $\mathrm{T} 1$ recognition, with three $\mathrm{T} 2$ Locations (Left, Center, Right) and five Lags $(1,2,4,6,7)$, revealed a main effect of T2 Location, $F(1,18)=11.257, \mathrm{MSE}=.004, \eta_{p}{ }^{2}=.387, P=$ .004 , and a main effect of Lag, $F(1,18)=5.735, \mathrm{MSE}=.002$, $\eta_{p}{ }^{2}=.242, P=.028$. Moreover, there was an interaction between T2 Location and Lag, $F(1,18)=13.288, M S E=$ $.003, \eta_{p}{ }^{2}=.425, P=.002$ (see Fig. 2a). Planned comparisons
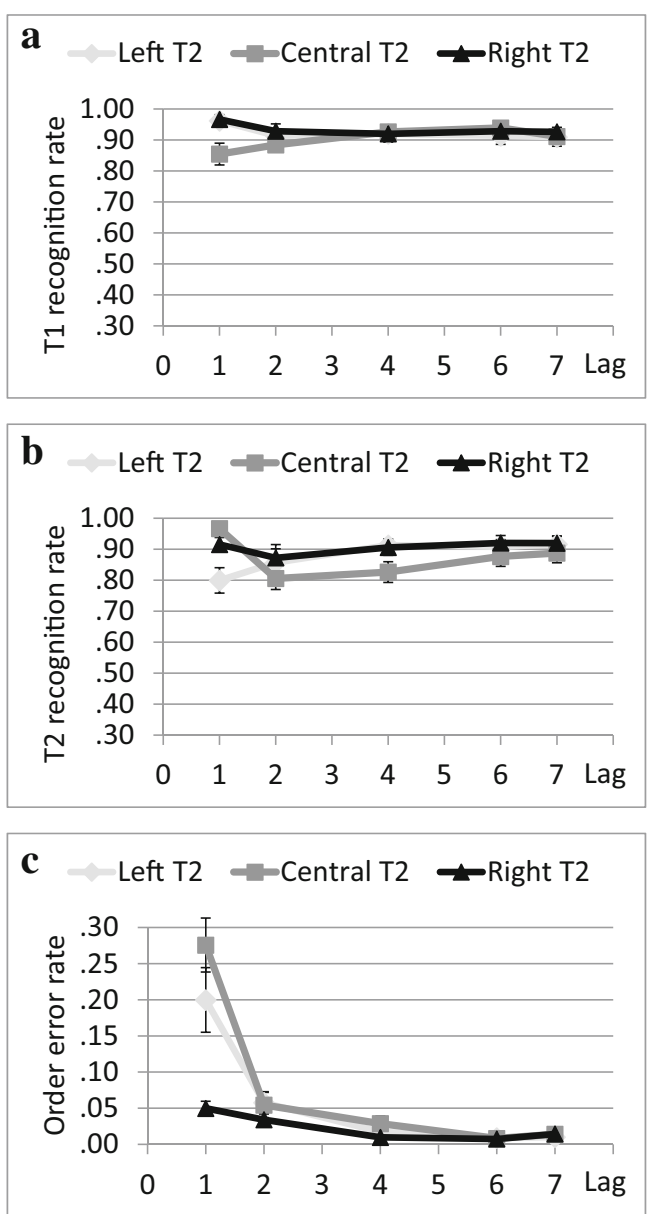

Fig. 2 a-c Experiment 1a. a T1 recognition rate, b T2| T1 recognition rate and $\mathbf{c} \mathrm{T} 1-\mathrm{T} 2$ order error rate as a function of $\mathrm{T} 2$ Location (Left, Center or Right) and T1-T2 Lag (1, 2, 4, 6, 7). Error bars 1 standard error (SE) 
that focused at lag 1 and 2 revealed that when followed by a central T2, T1 recognition did not differ between lag 1 and lag $2, t(18)=1.508, P=.149$. As our main interest was in leftright asymmetry, we performed a $2 \mathrm{X} 2$ ANOVA with two T2 Locations (Left, Right) and two Lags $(1,2)$. This analysis showed that when $\mathrm{T} 2$ did not follow on the center, T1 recognition was slightly higher at lag $1(M=.96, \mathrm{SE}=.01)$ than at lag $2(M=.92, \mathrm{SE}=.02), F(1,18)=7.288, \mathrm{MSE}=.005, \eta_{p}{ }^{2}=$ $.288, P=.015$. However, neither a significant effect was found for T2 Location, $F(1,18)=2.209$, MSE $=.001, \eta_{p}{ }^{2}=.109, P=$ .155 , nor did the Lag effect differ between left and right $\mathrm{T} 2$ presentation, $F<1$.

\section{T2| T1 recognition rate}

A repeated measures $3 \times 5$ ANOVA on conditional T2 recognition rate (T2 $\mid \mathrm{T} 1$ ), with three T2 Locations (Left, Center, Right) and five Lags $(1,2,4,6,7)$, revealed a marginally significant main effect of T2 Location, $F(1,18)=3.833$, $M S E=.007, \eta_{p}{ }^{2}=.176, P=.066$, and a main effect of Lag, $F(1,18)=5.662, M S E=.01, \eta_{p}{ }^{2}=.239, P=.029$. More interestingly, there was an interaction between T2 Location and Lag, $F(1,18)=20.913, M S E=.008, \eta_{p}^{2}=.537, P<$ .001 (see Fig. 2b). Central T2 presentation resulted in a typical U-shaped AB curve. As expected, central T2s showed a significant Lag-1 sparing, with $\mathrm{T} 2$ being better recognized at lag 1 than at lag $2, t(18)=4.61, P<.001$. In contrast, shifting T2 to the right or to the left resulted in much shallower curves.

As our main interest was in asymmetry in the $A B$, we carried out a $2 \times 2$ ANOVA with two T2 Locations (Left, Right) and two Lags $(1,2)$. This analysis revealed that while Lag had no significant effect, $F<1$, there was a significant effect of T2 Location, $F(1,18)=9.781$, MSE $=.008, \eta_{p}{ }^{2}=$ $.352, P=.006$, with $\mathrm{T} 2$ better recognized on the right $(M=$ $.89, \mathrm{SE}=.03)$ than on the left $(M=.83, \mathrm{SE}=.04)$. Moreover, the interaction of T2 Location and Lag was significant, $F$ $(1,18)=7.904, \mathrm{MSE}=.008, \eta_{p}{ }^{2}=.305, P=.012$. Planned comparisons revealed that that there was no Lag-1 sparing for both left and right T2s, $P$ values $>.212$. In contrast, comparing the effect of T2 Location in each lag revealed that while at lag 1 , right T2s were better recognized than left T2s, $t(18)=3.88$, $P=.001$, in lag 2, T2 Location (Left, Right) did not affect T2 recognition, $t<1$.

\section{T1-T2 order errors}

As order errors are expected to be found at lag 1 trials, our analysis focused on comparing order errors in lags 1 and 2. A repeated measures $3 \times 2$ ANOVA on T1-T2 order error rate calculated relative to the total number of trials where both targets were recognized (cf. Akyurek et al., 2012; Chun \& Potter, 1995), with three T2 Locations (Left, Center, Right) and two Lags $(1,2)$, revealed a main effect of T2 Location, $F$
$(1,18)=9.753, \mathrm{MSE}=.015, \eta_{p}{ }^{2}=.351, P=.006$, and a main effect of Lag, $F(1,18)=68.044, \mathrm{MSE}=.007, \eta_{p}{ }^{2}=.791, P<$ .001 . Moreover, there was an interaction between T2 Location and Lag, $F(1,18)=11.248, \mathrm{MSE}=.011, \eta_{p}{ }^{2}=.385, P=.004$ (see Fig. 2c). Further analysis revealed that for central T2s, order error rate was higher at lag 1 than at lag $2, t(18)=7.367$, $P<.001$. A $2 \times 2$ ANOVA with two T2 Locations (Left, Right) and two Lags $(1,2)$ revealed a significant Lag effect, $F(1,18)$ $=12.42, \mathrm{MSE}=.01, \eta_{p}{ }^{2}=.408, P=.002$, with more order errors in lag $1(M=.13, S E=.02)$ than in lag $2(M=.05, \mathrm{SE}=$ $.01)$. Moreover, we found a significant effect for T2 Location, $F(1,18)=9.753, \mathrm{MSE}=.015, \eta_{p}{ }^{2}=.351, P=.006$, with more order errors on the left $(M=.13, \mathrm{SE}=.03)$ than on the right $(M$ $=.04, S E=.01)$. Importantly, also a significant interaction was found, $F(1,18)=8.122$, MSE $=.009, \eta_{p}{ }^{2}=.311, P=.011$, with more order errors for left $\mathrm{T} 2 \mathrm{~s}$ than for right $\mathrm{T} 2 \mathrm{~s}$ at lag $1, t$ $(18)=3.183, P=.005$, but no asymmetry in order errors in lag 2, $t(18)=1.337, P=.198$.

To summarize, central $\mathrm{T} 2$ presentation resulted in a Ushaped $\mathrm{AB}$ curve, with Lag-1 sparing and pronounced amount of order errors at lag 1. Unlike Verlerger and colleagues' LVF advantage, we found an asymmetry on $\mathrm{T} 2$ recognition between right and left $\mathrm{T} 2 \mathrm{~s}$, demonstrating a right visual-field (RVF) advantage. While left T2 exhibited some of the characteristics of the $\mathrm{AB}$, right $\mathrm{T} 2 \mathrm{~s}$ did not demonstrate any. Interestingly, although no apparent Lag-1 sparing occurred for left T2s, left T2s demonstrated at lag 1 a high order error rate. In the same vein, lack of $\mathrm{AB}$ for right $\mathrm{T} 2 \mathrm{~s}$ was also indicated by the low order error rate for right $\mathrm{T} 2 \mathrm{~s}$ at lag 1 .

\section{Experiment 1b}

One might be concerned that the lack of Lag-1 sparing (and $\mathrm{AB}$ ) for right $\mathrm{T} 2$ could have resulted from a ceiling effect. To reduce the possibility of a ceiling effect for right $\mathrm{T} 2 \mathrm{~s}$, and to test the replicability of the RVF advantage, we ran the same experiment again, this time we reduced stimuli presentation duration to reduce targets' visibility, and introduced blank intervals in between items in order to keep the same stimulus-onset asynchrony (SOA) as in Exp. 1a.

\section{Method}

\section{Participants}

Twenty-three undergraduate students (five males, aged 20-31) from the Academic College of Tel-Aviv-Yaffo participated for class credit. All reported normal or corrected-to-normal vision. One participant was excluded from the analysis because of a low T1 recognition rate in one of the conditions, deviating below the group mean by more than 2.5 SDs. 
Apparatus and stimuli

Apparatus and stimuli were the same as in Exp. 1a.

\section{Procedure}

The same as Exp. 1a, except that $40 \mathrm{~ms}$ blank intervals were inserted between consecutive stimuli, and stimuli were presented only for $67 \mathrm{~ms}$, keeping an SOA of $107 \mathrm{~ms}$.

\section{Results and discussion}

\section{T1 recognition rate}

A repeated measures $3 \times 5$ ANOVA on T1 recognition, with three T2 Locations (Left, Center, Right) and five Lags (1, 2, 4, $6,7)$, revealed a main effect of T2 Location, $F(1,21)=6.933$, $\mathrm{MSE}=.003, \eta_{p}{ }^{2}=.243, P=.016$, and a marginally significant effect of Lag, $F(1,21)=4.3, \mathrm{MSE}=.003, \eta_{p}{ }^{2}=.17, P=.051$. Moreover, there was a significant interaction between T2 Location and Lag, $F(1,21)=26.373, \mathrm{MSE}=.002, \eta_{p}{ }^{2}=$ $.557, P<.001$ (see Fig. 3a). Planned comparisons that focused at lags 1 and 2 revealed that when followed by a central T2, T1 recognition rate was reduced in lag 1 compared to lag 2, $t$ (21) $=4.375, P<.001$. In contrast, a $2 \times 2$ ANOVA with two T2 Locations (Left, Right) and two Lags $(1,2)$, showed that when T2 did not follow on the center, T1 recognition was higher at lag $1(M=.96, \mathrm{SE}=.01)$ than at lag $2(M=.94, \mathrm{SE}=.01), F$ $(1,21)=4.608, \mathrm{MSE}=.001, \eta_{p}{ }^{2}=.18, P=.044$. However, neither a significant effect was found for T2 Location, $F(1,21)$ $=1.115, \mathrm{MSE}=.001, \eta_{p}{ }^{2}=.05, P=.303$, nor did the lag effect differ between left and right T2 presentation, $F<1$.

\section{T2| T1 recognition rate}

A repeated measures $3 \times 5$ ANOVA on conditional T2 recognition rate (T2| T1), with three $\mathrm{T} 2$ Locations (Left, Center, Right) and five Lags (1, 2, 4, 6, 7), revealed a significant effect of T2 Location, $F(1,21)=3.83$, MSE $=.013, \eta_{p}{ }^{2}=.216, P=$ .025 , and a main effect of Lag, $F(1,21)=16.608, \mathrm{MSE}=.016$, $\eta_{p}{ }^{2}=.442, P=.001$. Reproducing Exp. 1, there was an interaction between T2 Location and Lag, $F(1,21)=38.238$, MSE $=.009, \eta_{p}{ }^{2}=.645, P<.001$ (see Fig. 3b). Central T2 presentation resulted in a typical U-shaped AB curve. Accordingly, central T2s showed a Lag-1 sparing, with T2 being better recognized at lag 1 than at lag $2, t(21)=5.697, P<.001$. In contrast, shifting $\mathrm{T} 2$ to the right or to the left resulted in much shallower curves.

As in Exp. 1a, we further carried a $2 \times 2$ ANOVA with two T2 Locations (Left, Right) and two Lags $(1,2)$. This analysis reproduced Exp. 1a pattern, revealing that while Lag had no significant effect, $F(1,21)=1.188, \mathrm{MSE}=.011, \eta_{p}{ }^{2}=.054, P$ $=.288$, there was a marginally significant effect of T2
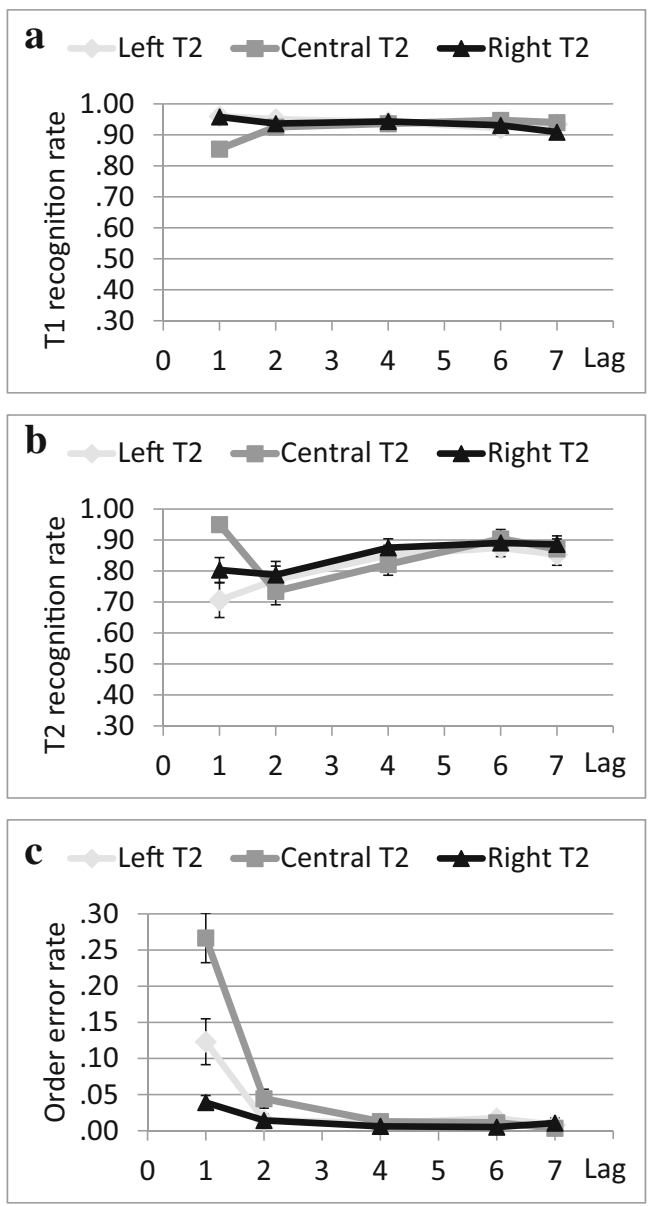

Fig. 3 a-c Experiment 1b. a T1 recognition rate, b T2| T1 recognition rate and $\mathbf{c} \mathrm{T} 1-\mathrm{T} 2$ order error rate as a function of T2 Location (Left, Center or Right) and T1-T2 Lag (1, 2, 4, 6, 7). Error bars $1 \mathrm{SE}$

Location, $F(1,21)=3.34$, MSE $=.021, \eta_{p}{ }^{2}=.137, P=$ .082 , with T2 slightly better recognized on the right $(M=$ $.80, \mathrm{SE}=.04)$ than on the left $(M=.74, \mathrm{SE}=.05)$. Importantly, the interaction of T2 Location and Lag was significant, $F(1,21)=6.958, \mathrm{MSE}=.005, \eta_{p}{ }^{2}=.249, P=.015$. Following Exp. 1a, our planned comparisons focused on leftright asymmetry and showed that while at lag 1 right $\mathrm{T} 2 \mathrm{~s}$ were better recognized than left T2s, $t(21)=2.453, P=.023$, in lag $2 \mathrm{~T} 2$ recognition on the right and left did not differ, $t<1$.

\section{T1-T2 order errors}

One participant was excluded from the following analysis due to having less than five trials in which both T1 and T2 were recognized at least in one of the conditions, as such a low number of trials might artificially inflate order error rate. A repeated measures $3 \times 2$ ANOVA on T1-T2 order error rate calculated relative to the total number of trials where both targets were recognized, with three T2 Locations (Left, Center, Right) and two Lags $(1,2)$, revealed a main effect of 
T2 Location, $F(1,20)=34.945, \mathrm{MSE}=.009, \eta_{p}{ }^{2}=.636, P<$ .001 , and a main effect of Lag, $F(1,20)=43.265, \mathrm{MSE}=.01$, $\eta_{p}{ }^{2}=.684, P<.001$. Moreover, there was an interaction between T2 Location and Lag, $F(1,20)=18.169$, MSE $=.009$, $\eta_{p}{ }^{2}=.476, P<.001$ (see Fig. 3c). Further analysis revealed that, for central T2s, order error rate was higher at lag 1 than at lag $2, t(20)=6.342, P<.001$. A $2 \times 2$ ANOVA with two T2 Locations (Left, Right) and two Lags $(1,2)$ revealed a significant effect for T2 Location, $F(1,20)=7.522, \mathrm{MSE}=.005, \eta_{p}{ }^{2}$ $=.273, P=.013$, with more order errors on the left $(M=.07$, $\mathrm{SE}=.02)$ than on the right $(M=.03, \mathrm{SE}=.01)$. There was also a significant Lag effect, $F(1,20)=13.926, \mathrm{MSE}=.007, \eta_{p}{ }^{2}=$ $.41, P=.001$, with more order errors in lag $1(M=.08, \mathrm{SE}=$ $.02)$ than in lag $2(M=.02, \mathrm{SE}=.01)$. Importantly, a significant interaction was found, $F(1,20)=10.472, \mathrm{MSE}=.003$, $\eta_{p}{ }^{2}=.344, P=.004$, with more order errors for left T2s than for right T2s at lag $1, t(20)=3.095, P=.006$, but no asymmetry in order errors in lag $2, t<1$.

Exp. $1 \mathrm{~b}$ replicated the findings of Exp. 1a, showing they were not due to a ceiling effect. Although performance for T2 recognition was reduced in lags 1 and 2, an RVF advantage at lag 1 emerged. As in Exp. 1a, better recognition of right T2s at lag 1 was accompanied by close to zero order errors. In contrast, left T2s demonstrated a pronounced level of order errors.

Considering T1-T2 order errors for central T2s, both experiments replicate previous reports of a high error rate (e.g., Akyürek et al., 2008, 2012; Chun \& Potter, 1995, Exp. 5; Hilkenmeier et al., 2012; Olivers et al., 2011; Shih, 2000). When considering order error rate of shifted T2s, a further support for an RVF advantage was found. Although left T2s did not demonstrate a Lag-1 sparing in the traditional sense (improved $\mathrm{T} 2$ recognition in lag 1 ), they showed a high order error rate, significantly higher than the order error rate of right $\mathrm{T} 2 \mathrm{~s}$. The higher order error rate on left T2s compared to right T2s is similar to the results pattern of Shih (2000) (although not statistically verified in her study), who used a dual-stream RSVP procedure and reported a higher order error rate for left $\mathrm{T} 2 \mathrm{~s}$ (preceded by right $\mathrm{T} 1$ ) than for right $\mathrm{T} 2 \mathrm{~s}$ (preceded by left T1).

Previous studies that reported asymmetry in the AB found an LVF advantage (reviewed in Verleger \& Smigasiewicz, 2015 ) but did not measure order errors. Verleger and Smigasiewicz (2015) suggested that their LVF advantage resulted from the right hemisphere being superior in directing attention reactively to salient events. However, our Exp. 1a and $1 \mathrm{~b}$ undermines this assertion as bottom up attention triggered by the abrupt onset of T2 lead to RVF advantage.

The aim of Experiment 2 was to test whether RVF advantage found in our study indeed depends on T2 abrupt onset. Our experiments and the studies of Verleger and colleagues differed in various additional aspects (e.g., T1 location, and whether there was a task switch between T1 and T2), rather than only whether T2 attracted attention in a bottom-up manner. Therefore, in Exp. 2 we tried to cancel RVF advantage by removing the abrupt onset of $\mathrm{T} 2$.

\section{Experiment 2}

The purpose of Exp. 2 was to test whether RVF advantage on T2 recognition in Exp. 1a/1b depended on the abrupt onset of T2. To achieve this aim, in Exp. 2, T1 and T2 were embedded within three streams of stimuli, with $\mathrm{T} 1$ always presented on the central stream and T2 presented on the left, the center or the right stream.

\section{Method}

\section{Participants}

Twenty undergraduate students (one male, aged 22-28 years) from the Academic College of Tel-Aviv-Yaffo participated for class credit. All reported normal or corrected-to-normal vision. None of the participants participated in Exp. 1a or $1 \mathrm{~b}$.

\section{Apparatus and stimuli}

Apparatus and stimuli were the same as in Exp. 1a.

\section{Procedure}

The procedure was the same as in Exp. 1a except that in Exp. 2 we used three streams of distractor letters from the very beginning of each trial. As in Exp. 1a, T1 was always presented on the central stream, and T2 was presented on the left, on the center, or on the right stream (see Fig. 4). As in Exp. 1a, all stimuli were presented for $107 \mathrm{~ms}$ without blank inter-trial intervals.

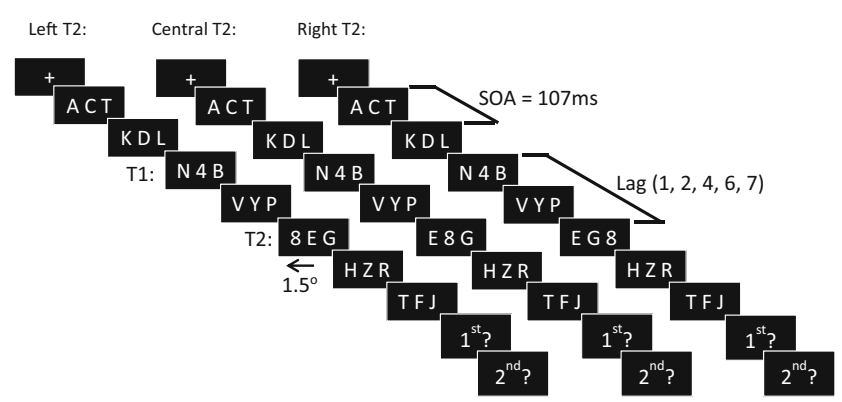

Fig. 4 Experiment 2: Three-stream RSVP with a digit T1 embedded in a stream of letter distractors presented at the central position, surrounded by two streams of letter distractors. T2 and the following distractors were presented at the left, center or right position, with continuous streams of distractors in the other two positions. T2 could lag behind T1 in the 1st, 2nd, 4th, 6th or 7th position. Lag 2 is illustrated. 


\section{Results and discussion}

\section{T1 recognition rate}

A repeated measures $3 \times 5$ ANOVA on T1 recognition, with three T2 Locations (Left, Center, Right) and five Lags (1, 2, 4, $6,7)$, revealed a main effect of T2 Location, $F(1,19)=5.955$, MSE $=.006, \eta_{p}{ }^{2}=.239, P=.025$, and a main effect of Lag, $F$ $(1,19)=19.386, \mathrm{MSE}=.004, \eta_{p}{ }^{2}=.505, P<.001$. Moreover, there was an interaction between T2 Location and Lag, $F$ $(1,19)=36.096, \mathrm{MSE}=.003, \eta_{p}{ }^{2}=.655, P<.001($ see Fig. 5a). Planned comparisons that focused at lags 1 and 2 revealed that when followed by a central $\mathrm{T} 2, \mathrm{~T} 1$ recognition rate was reduced in lag 1 compared to lag2, $t(19)=4.46, P<$ .001 . In contrast, a $2 \times 2$ ANOVA with two T2 Locations (Left, Right) and two Lags $(1,2)$, showed that when T2 did not follow on the center, T1 recognition was not affected by Lag, $F<1$, by T2 Location, $F(1,19)=1.377, M S E=.006$, $\eta_{p}{ }^{2}=.068, P=.255$, nor did the two factors interact, $F<1$.
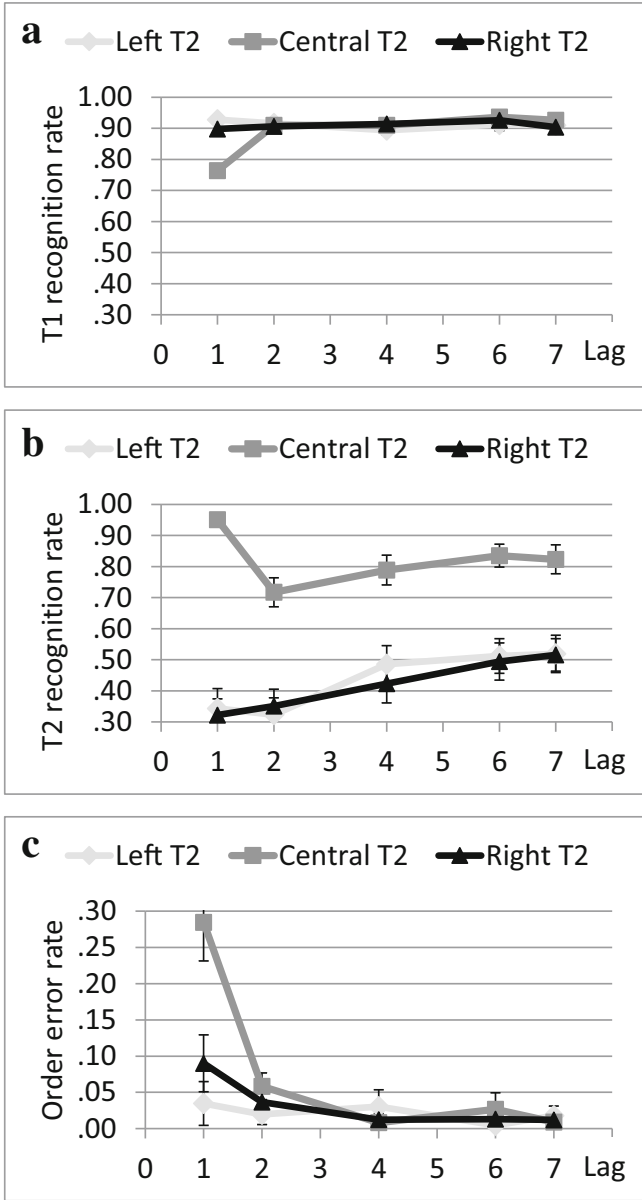

Fig. 5 a-c Experiment 2. a T1 recognition rate, b T2| T1 recognition rate and $\mathbf{c}$ T1-T2 order error rate as a function of T2 Location (Left, Center or Right) and T1-T2 Lag (1, 2, 4, 6, 7). Error bars $1 \mathrm{SE}$
T2| T1 recognition rate

A repeated measures $3 \times 5$ ANOVA on conditional T2 recognition rate (T2| T1), with three T2 Locations (Left, Center, Right) and five Lags $(1,2,4,6,7)$, revealed a main effect of T2 Location, $F(1,19)=70.043, \mathrm{MSE}=.148, \eta_{p}{ }^{2}=.401, P<$ .001 , and a main effect of Lag, $F(1,19)=29.359, M S E=.01$, $\eta_{p}{ }^{2}=.607, P<.001$. Importantly, there was an interaction between T2 Location and $\operatorname{Lag}, F(1,19)=38.135$, MSE $=$ $.008, \eta_{p}{ }^{2}=.667, P<.001$ (see Fig. 5b). Central T2 presentation resulted in a typical U-shaped AB curve. Accordingly, central T2s showed a significant Lag-1 sparing, with T2 being better recognized at lag 1 than at lag $2, t(19)=6.124, P<$ .001 . In contrast, shifting T2 to the right or to the left resulted in much worse performance in all lags and in monotonic, rather than U-shaped, curves. As in Exp. 1, we further carried a $2 \times 2$ ANOVA with two T2 Locations (Left, Right) and two Lags $(1,2)$. This analysis revealed that both Lag and T2 Location had no significant effects, $F$ values $<1$, nor did they interact, $F(1,19)=1.579, \mathrm{MSE}=.008, \eta_{p}{ }^{2}=.077, P=.224$.

\section{T1-T2 order errors}

Nine participants were excluded from the following analysis due to having less than five trials in which both T1 and T2 were recognized at least in one of the conditions. A repeated measures $3 \times 2$ ANOVA on T1-T2 order error rate calculated relative to the total number of trials where both targets were recognized, with three T2 Locations (Left, Center, Right) and two Lags $(1,2)$, revealed a main effect of T2 Location, $F$ $(1,10)=15.559, \mathrm{MSE}=.015, \eta_{p}{ }^{2}=.609, P=.003$, and a main effect of Lag, $F(1,10)=12.119$, MSE $=.013, \eta_{p}{ }^{2}=.548, P=$ .006. Moreover, there was an interaction between T2 Location and Lag, $F(1,10)=12.839, \mathrm{MSE}=.011, \eta_{p}{ }^{2}=.562, P=.005$ (see Fig. 5c). Further analysis revealed that for central T2s, order error rate was higher at lag 1 than at lag $2, t(10)=4.822$, $P=.001$. A $2 \times 2$ ANOVA with two T2 Locations (Left, Right) and two Lags $(1,2)$ revealed that neither T2 Location, $F(1,10)$ $=2.081, \mathrm{MSE}=.007, \eta_{p}{ }^{2}=.172, P=.18$, nor Lag had an effect on order errors, $F(1,10)=1.118, \mathrm{MSE}=.012, \eta_{p}{ }^{2}=$ $.101, P=.315$, and that the two factors did not interact, $F<1$.

As expected, elimination of the abrupt onset of T2 in Exp. 2 erased the RVF advantage. Spatially shifted T2s showed a monotonic AB curve, without Lag-1 sparing and no order errors, with the left and right $\mathrm{AB}$ curves being practically identical. Note, that eliminating bottom-up saliency, forces participants to rely on top-down selection. It seems that relying solely on top-down attention diminished RVF advantage.

Exp. 2 generated a completely different pattern of results from Exp. 1a and 1b. However, in comparison to Exp. 1a and $1 b$, not only that shifted-T2s did not abruptly onset (as they were embedded in a stream of distractors), they also cooccurred with two distractors. In Exp. 3 we reintroduced 
abrupt onset of shifted-T2s, but kept the competing distractors. This forced participants to rely on top-down selection but still allows them to benefit from bottom-up saliency. We asked whether the reintroduction of bottom-up saliency is enough for RVF advantage to reemerge.

\section{Experiment 3}

In Exp. 3, each RSVP started only with one central stream in which T1 was embedded. Then, T2 was presented in the left, central or right location, co-occurring with two distractors. This resulted in T1 being masked at lag-1 trials also when T2 was shifted, unlike in Exp. 1a and 1b, but similarly to Exp. 2. As in Exp. 2, three streams of distractors followed, backward masking T2 as well as its competing distractors. Thus, shifted-T2s benefited from abrupt onset but were hindered by two other counterparts. ${ }^{2}$ This allowed us to examine whether abrupt onset by itself is sufficient for RVF advantage to emerge, even when participants have to rely on top-down selection.

\section{Method}

\section{Participants}

Twenty-two undergraduate students (two males, aged 19-36 years) from the Academic College of Tel-Aviv-Yaffo participated for class credit. All reported normal or corrected-tonormal vision. None of the participants participated in the previous experiments. Two participants were excluded from the analysis because of a low T1 recognition rate in lag 1 or in additional lags, deviating below the group mean by more than 2.5 SDs.

\section{Apparatus and stimuli}

Apparatus and stimuli were the same as in Exps. 1 and 2.

\section{Procedure}

The procedure was the same as in Exp. 2, except for one change: as in Exp. 1, each trial started with only one central RSVP stream, including the letter distractors and the digit T1 (see Fig. 6). This change resulted in the side positions not being marked in advance before $\mathrm{T} 2$ presentation. In this

\footnotetext{
${ }^{2}$ Note that Yantis and Johnson (1990) and Yantis and Jones (1991) found that, in a visual search task, abrupt onset of several stimuli or one stimulus demonstrated a similar pattern of results. Yantis (1993) concluded that, when several stimuli abruptly onset simultaneously, all the onset stimuli are immediately tagged as high priority compared to the non-onset elements. Thus, following their conceptualization, in the current experiment shifted-T2s are considered as abruptly onset.
}

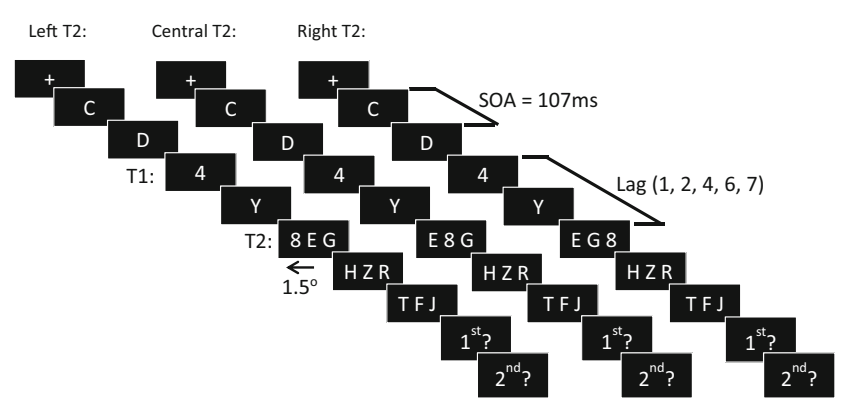

Fig. 6 Experiment 3. One-stream RSVP with a digit T1 embedded in a stream of letter distractors, all presented at central position. T2 and the following distractors were presented at the left, center or right position, with continuous streams of letter distractors in the other two positions. T2 could lag behind $\mathrm{T} 1$ in the 1 st, 2 nd, 4 th, 6 th or 7 th position. Lag 2 is illustrated

way, T2 presentation on the side streams was an abrupt onset, but it co-occurred with two letter distractors. Note that unlike Exp. 1, but similarly to Exp. 2, at lag 1, T1 was backwardmasked not only when $\mathrm{T} 2$ was presented on the center but also when T2 was presented on either side stream because it was followed by a distractor letter.

\section{Results and discussion}

\section{T1 Recognition rate}

A repeated measures $3 \times 5$ ANOVA on T1 recognition, with three T2 Locations (Left, Center, Right) and five Lags (1, 2, 4, $6,7)$, revealed a marginally significant effect for T2 Location, $F(1,19)=4.15, \mathrm{MSE}=.002, \eta_{p}{ }^{2}=.179, P=.056$, and a main effect of Lag, $F(1,19)=23.444, \mathrm{MSE}=.005, \eta_{p}{ }^{2}=.552, P<$ .001 . Moreover there was an interaction between T2 Location and Lag, $F(1,19)=18.374, \mathrm{MSE}=.003, \eta_{p}{ }^{2}=.492, P<.001$ (see Fig. 7a). Simple effects analysis revealed that when T2 followed on the center, $\mathrm{T} 1$ recognition rate was reduced in lag 1 compared to lag $2, t(19)=4.042, P=.001$. A $2 \times 2$ ANOVA with two T2 Locations (Left, Right) and two Lags (1, 2), showed that when $\mathrm{T} 2$ did not follow on the center, $\mathrm{T} 1 \mathrm{recog}-$ nition was lower at lag $1(M=.88, \mathrm{SE}=.03)$ than at lag $2(M=$ $.95, \mathrm{SE}=.02), F(1,19)=18.255, \mathrm{MSE}=.006, \eta_{p}{ }^{2}=.49, P<$ .001 . There was also an effect for T2 Location, $F(1,19)=$ 5.444, MSE $=.003, \eta_{p}{ }^{2}=.223, P=.031$, with T1 being better recognized when $\mathrm{T} 2$ followed on the right $(M=.93, \mathrm{SE}=.02)$ than on the left $(M=.91, \mathrm{SE}=.03)$. Nevertheless, the two factors did not interact, $F<1$.

\section{T2| T1 recognition rate}

A repeated measures $3 \times 5$ ANOVA on conditional T2 recognition rate (T2 $\mid \mathrm{T} 1)$, with three $\mathrm{T} 2$ Locations (Left, Center, Right) and five Lags $(1,2,4,6,7)$, revealed no significant effect of T2 Location, $F(1,19)=2.335, \mathrm{MSE}=.054, \eta_{p}{ }^{2}=$ $.109, P=.143$. In contrast, there was a main effect of Lag, 

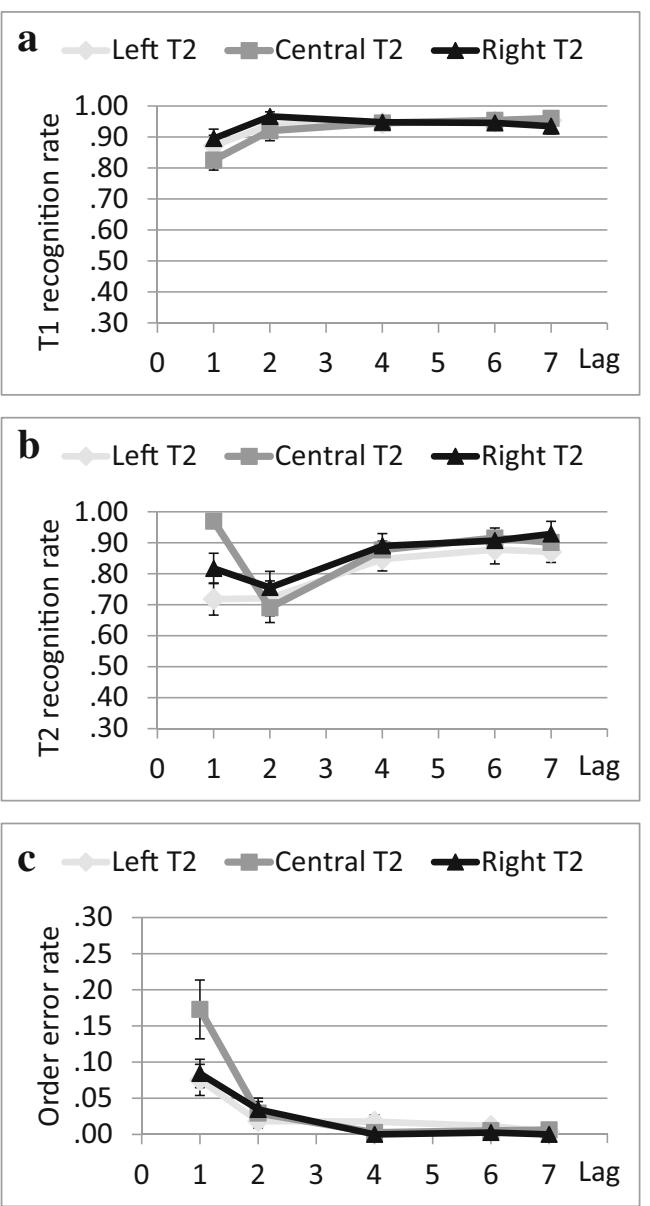

Fig. 7 a-c Experiment 3. a T1 recognition rate, b T2 $\mid \mathrm{T} 1$ recognition rate and $\mathbf{c} \mathrm{T} 1-\mathrm{T} 2$ order error rate as a function of T2 Location (Left, Center or Right) and T1-T2 Lag $(1,2,4,6,7)$. Error bars $1 \mathrm{SE}$

$F(1,19)=34.501, \mathrm{MSE}=.017, \eta_{p}{ }^{2}=.645, P<.001$. More interestingly, there was an interaction between T2 Location and Lag, $F(1,19)=22.229, \mathrm{MSE}=.006, \eta_{p}{ }^{2}=.539, P<$ .001 (see Fig. 7b). Central T2 presentation resulted in a typical U-shaped AB curve. Accordingly, central T2s showed a significant Lag-1 sparing, with $\mathrm{T} 2$ being better recognized at lag 1 than at lag $2, t(19)=5.74, P<.001$. A $2 \times 2$ ANOVA with two T2 Locations (Left, Right) and two Lags $(1,2)$ revealed that neither Lag, $F(1,19)=1.487, \mathrm{MSE}=.016, \eta_{p}{ }^{2}=.073, P=$ .238 , nor T2 Location had a significant effect of T2 recognition, $F(1,19)=1.953, \mathrm{MSE}=.04, \eta_{p}{ }^{2}=.093, P=.178$. Nevertheless, the two factors showed a marginally significant interaction, $F(1,19)=4.36, \mathrm{MSE}=.005, \eta_{p}{ }^{2}=.187$, $P=.051$. Planned comparisons revealed that while at lag 1 , right $\mathrm{T} 2 \mathrm{~s}$ were better recognized than left T2s, $t$ (19) $=$ 2.211, $P=.04$, in lag 2, T2 Location (Left, Right) did not affect $\mathrm{T} 2$ recognition, $t<1$. In fact, Lag-1 sparing was significant for right T2s, $t(19)=2.475, P=.023$, with lag $1 \mathrm{~T} 2 \mathrm{~s}$ being better recognized than lag $2 \mathrm{~T} 2 \mathrm{~s}$, but no Lag-1 sparing was found for left T2s, $t<1$.

\section{T1-T2 order errors}

Two participants were excluded from the following analysis due to having less than five trials in which both T1 and T2 were recognized at least in one of the conditions. A repeated measures $3 \times 2$ ANOVA on T1-T2 order error rate calculated relative to the total number of trials where both targets were recognized, with three T2 Locations (Left, Center, Right) and two Lags $(1,2)$, revealed a marginally significant effect for T2 Location, $F(1,17)=4.155, \mathrm{MSE}=.013, \eta_{p}{ }^{2}=.196, P=.057$, and a main effect for $\mathrm{Lag}, F(1,17)=20.219, \mathrm{MSE}=.009, \eta_{p}{ }^{2}$ $=.543, P<.001$. Moreover, there was a marginally significant interaction between T2 Location and Lag, $F(1,17)=3.789$, MSE $=.013, \eta_{p}{ }^{2}=.182, P=.068$ (see Fig. $7 \mathrm{c}$ ). Planned comparisons revealed that for central $\mathrm{T} 2 \mathrm{~s}$, order error rate was higher at lag 1 than at lag $2, t(17)=3.107, P=.006$. A $2 \times 2$ ANOVA with two T2 Locations (Left, Right) and two Lags $(1,2)$ revealed a significant effect for Lag, $F(1,17)=$ 17.33, $\mathrm{MSE}=.003, \eta_{p}{ }^{2}=.505, P=.001$, with more order errors in lag $1(M=.08, \mathrm{SE}=.02)$ than at lag $2(M=.03, \mathrm{SE}=$ .01). However, neither T2 Location nor the interaction of T2 Location and Lag were significant, both $F$ values $<1$.

In contrast to Exp. 1, where order errors were more likely for left than for right T2s, in Exp. 3, order error rate did not differ between left and right T2s. Indeed, a $2 \times 2$ ANOVA with T2 Location (Left, Right) and Experiment $(1,3)$ revealed a significant interaction, $F(1,35)=8.964, \mathrm{MSE}=.013, \eta_{p}{ }^{2}=$ $.204, P=.005$, with higher rate of order errors on left T2s than right T2s in Exp. 1 and no difference between error rate on left and right T2s in Exp. 3. In fact, left T2s showed significantly more order errors in Exp. 1 than in Exp. 3, $t(35)=2.461, P=$ .019 , while for right $\mathrm{T} 2 \mathrm{~s}$, order error rate did not differ between the two experiments, $t(35)=1.596, P=.119$.

In Exp. 3 the RVF advantage reemerged. For right T2s a Lag-1 sparing surfaced. However, this advantage was not accompanied by a difference in order errors, as the rate of such errors was similarly low for left and right T2s. As noted earlier, however, an additional difference exists between Exp. 3 and Exp. 1. While in Exp. 3 T1 was masked at lag 1 trials, it was not masked in Exp. 1. Thus, it is unclear whether the lack of order error asymmetry in Exp. 3 is the result of increased top-down selection requirements or the result of $\mathrm{T} 1$ being masked in lag 1 trials. To address this problem, in Exp. 4 shifted T2s were presented in conditions of abrupt onset (as in Exp. 1 and 3); however, T1 was masked in lag 1 trials, similarly to Exp. 3.

\section{Experiment 4}

Exp. 4 replicated Exp. 1a, with the exception that at lag 1 trials, spatially shifted T2s were presented concurrently with a central distractor that acted as a T1-mask. This resulted in T1 
being masked also at lag 1 trials, unlike in Exp. 1, but similarly to Exp. 2 and 3. Thus, shifted-T2s benefited from abrupt onset and required less top-down attention to be identified, compared to Exp. 3, where a distractor abruptly onset concurrently with $\mathrm{T} 2$.

\section{Method}

\section{Participants}

Sixteen undergraduate students (two males, aged 21-25 years) from the Academic College of Tel-Aviv-Yaffo participated for class credit. All reported normal or corrected-to-normal vision. None of the participants participated in the previous experiments.

\section{Apparatus and stimuli}

Apparatus and stimuli were the same as in the previous experiments.

\section{Procedure}

The procedure was the same as in Exp. 1a, except that at lag 1 trials, spatially shifted $\mathrm{T} 2 \mathrm{~s}$ co-occurred with a central letter distractor that masked $\mathrm{T} 1$.

\section{Results and discussion}

\section{T1 recognition rate}

A repeated measures $3 \times 5$ ANOVA on T1 recognition, with three T2 Locations (Left, Center, Right) and five Lags (1, 2, 4, $6,7)$, revealed no effect for T2 Location, $F(1,15)=1.79$, MSE $=.001, \eta_{p}{ }^{2}=.107, P=.201$, but a main effect of Lag, $F(1,15)$ $=10.75, \mathrm{MSE}=.004, \eta_{p}{ }^{2}=.417, P=.005$. In this experiment, however, the interaction between T2 Location and Lag was not significant, $F(1,15)=2.05, \mathrm{MSE}=.002, \eta_{p}{ }^{2}=.138, P=$ .142 (see Fig. 8a). Simple effects analysis revealed that when $\mathrm{T} 2$ followed on the center, $\mathrm{T} 1$ recognition rate was reduced in lag 1 compared to lag $2, t(15)=3.496, P=.003$. A $2 \times 2$ ANOVA with two T2 Locations (Left, Right) and two Lags (1, 2 ), showed that when $\mathrm{T} 2$ did not follow on the center, T1 recognition was lower at lag $1(M=.93, \mathrm{SE}=.02)$ than at lag $2(M=.97, \mathrm{SE}=.01), F(1,15)=8.29, \mathrm{MSE}=.003, \eta_{p}{ }^{2}=$ $.356, P=.011$. However, T2 Location had no effect, $F<1$, nor did the two factors interact, $F(1,15)=.11, \mathrm{MSE}=.004, \eta_{p}{ }^{2}=$ $.007, P=.749$.

\section{T2| T1 recognition rate}

A repeated measures $3 \times 5$ ANOVA on conditional T2 recognition rate (T2| T1), with three T2 Locations (Left, Center,
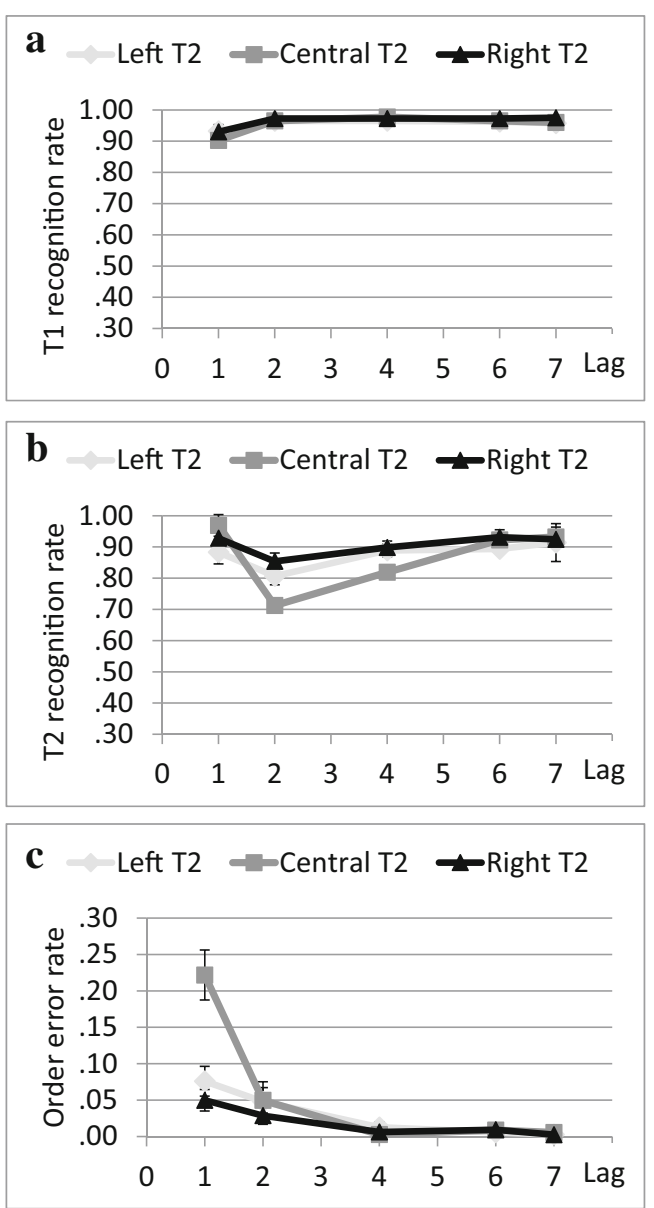

Fig. 8 a-c Experiment 4. a T1 recognition rate, b T2| T1 recognition rate and $\mathbf{c} \mathrm{T} 1-\mathrm{T} 2$ order error rate as a function of T2 Location (Left, Center or Right) and T1-T2 Lag (1, 2, 4, 6, 7). Error bars $1 \mathrm{SE}$

Right) and five Lags $(1,2,4,6,7)$, revealed only a marginally significant effect of T2 Location, $F(1,15)=3.519$, MSE $=.01$, $\eta_{p}{ }^{2}=.19, P=.08$. There was a main effect of Lag, $F(1,15)=$ $10.103, \mathrm{MSE}=.022, \eta_{p}{ }^{2}=.402, P=.006$. More interestingly, there was an interaction between T2 Location and Lag, $F$ $(1,15)=18.496, \mathrm{MSE}=.007, \eta_{p}{ }^{2}=.552, P=.001(\mathrm{see}$ Fig. 8 b). Central T2 presentation resulted in a typical Ushaped AB curve. Accordingly, central T2s showed a significant Lag-1 sparing, with $\mathrm{T} 2$ being better recognized at lag 1 than at lag $2, t(15)=4.00, P=.001$. A $2 \times 2$ ANOVA with two T2 Locations (Left, Right) and two Lags $(1,2)$ revealed a significant effect of Lag, $F(1,15)=5.62, \mathrm{MSE}=.016, \eta_{p}{ }^{2}=$ $.273, P=.032$, with $\mathrm{T} 2$ being better recognized at lag $1(M=$ $.91, \mathrm{SE}=.02)$ than at lag $2(M=.83, \mathrm{SE}=.04) . \mathrm{T} 2$ Location had a marginally significant effect on $\mathrm{T} 2$ recognition, $F(1,15)$ $=4.034, \mathrm{MSE}=.008, \eta_{p}{ }^{2}=.212, P=.063$, with T2 showing a tendency of being better recognized on the right $(M=.89$, SE $=.03)$ than on the left $(M=.85, \mathrm{SE}=.04)$. This time, however, the two factors did not interact, $F<1$. Although the interaction was not significant, we ran a planned comparisons analysis, 
which revealed that at lag 1 trials, right $\mathrm{T} 2 \mathrm{~s}$ were better recognized than left T2s, $t(15)=2.65, P=.018$. At lag 2 trials, however, T2 recognition did not differ between right and left, $t$ $(15)=1.11, P=.283$. Interestingly, while Lag-1 sparing was significant for right T2s, $t(15)=4.733, P=.006$, with lag 1 $\mathrm{T} 2 \mathrm{~s}$ being better recognized than lag $2 \mathrm{~T} 2 \mathrm{~s}$, left T2s did not demonstrate a significant Lag-1 sparing, $t(15)=1.444, P=$ .169 .

\section{T1-T2 order errors}

A repeated measures $3 \times 2$ ANOVA on T1-T2 order error rate calculated relative to the total number of trials where both targets were recognized, with three T2 Locations (Left, Center, Right) and two Lags $(1,2)$, revealed a main effect for Lag, $F(1,15)=12.723$, MSE $=.01, \eta_{p}{ }^{2}=.459, P=$ .003 , with a higher rate of order errors at lag $1(M=.12, \mathrm{SE}$ $=.02)$ than at lag $2(M=.04, \mathrm{SE}=.02)$. There was also a significant effect for T2 Location, $F(1,15)=21.106$, MSE = $.007, \eta_{p}{ }^{2}=.585, P<.001$, with a higher order error rate for central T2s. Importantly, there was a significant interaction between T2 Location and Lag, $F(1,15)=21.077$, MSE = $.006, \eta_{p}{ }^{2}=.584, P<.001$ (see Fig. 8c). Planned comparisons revealed that for central $\mathrm{T} 2 \mathrm{~s}$, order error rate was higher at lag 1 than at lag $2, t(15)=5.443, P<.001$. In contrast, a $2 \times 2$ ANOVA with two T2 Locations (Left, Right) and two Lags (1, 2) revealed neither an effect for Lag or for T2 Location, nor did the two factors interact, all $P$ values $>.115$.

Exp. 4 replicated the findings of Exp. 3, with an RVF advantage at lag 1 trials. Moreover, for right T2s a Lag-1 sparing surfaced, but, as in Exp. 3, this advantage was not accompanied by a difference in order error rate, as it was similarly low for left and right T2s. Thus, Exp. 4 demonstrated that order error elimination is not related to top-down requirements but is the result of T1-mask.

\section{General discussion}

Our experiments examined the effect of bottom-up attention on the AB. We used abrupt onset to manipulate bottom-up attention. In Exp. 1, we used one stream through the whole RSVP, resulting in abrupt onset for shifted T2s. This yielded an RVF advantage for shifted $\mathrm{T} 2 \mathrm{~s}$, with better recognition of right $\mathrm{T} 2 \mathrm{~s}$ compared to left $\mathrm{T} 2 \mathrm{~s}$ at lag 1 . This RVF advantage manifested itself also in a smaller order error rate for right $\mathrm{T} 2 \mathrm{~s}$ than for left T2s at lag 1. In Exp. 2, T2 was embedded in one of three streams, thus precluding the abrupt onset it benefited from in Exp. 1, requiring participants to rely on top-down selection. This caused shifted T2s to exhibit symmetrical monotonic curves with neither Lag-1 sparing nor order errors at lag 1. In Exp. 3, when shifted T2s abruptly onset with two competing distractors, an RVF advantage reemerged for T2 recognition, this time accompanied by Lag-1 sparing. However, there was no RVF advantage on order errors, as they occurred symmetrically at lag 1 , albeit on a lower rate than exhibited for left T2s in Exp. 1. Experiment 4 was a replication of Exp. 1, with the exception that at lag-1 trials, shifted T2s co-occurred with a T1-mask. Similarly to Exp. 3, shifted T2s showed an RVF advantage, and order errors were eliminated on both sides. Note that across the four experiments the possibility to rely on bottom-up attention was complemented by different levels of top-down selection requirements. Once bottom-up saliency could be utilized, RVF advantage emerged. However, order error rate was also affected by T1 masking. Namely, it reduced order errors on left T2s trials, leading to a similarly lower error rate for left and right T2s.

The existing theoretical frameworks accounting for Lag-1 sparing and order errors do not relate to spatial shifts of T2, nor to asymmetry in spatial-shift effects. We will present two current models of the $\mathrm{AB}$ and discuss their ability to reconcile the asymmetrical pattern of results obtained in Exp. 1. According to the Boost and Bounce theory (Olivers \& Meeter, 2008), T1 triggers a boost of attention, which gradually builds-up and peaks at about $100 \mathrm{~ms}$. If a distractor follows T1, attention is inhibited or bounced and AB occurs. In the case of lag 1, T2 benefits from the gradual attentional boost and reaches consciousness prior to T1 (e.g., Olivers, Scharlau, \& Hilkenmeier, 2011; Hilkenmeier, Olivers, \& Scharlau, 2012). Thus, T1-T2 order errors are caused by prior entry of T2 to consciousness. According to this framework, if $\mathrm{T} 2$ is presented at a different location, it should not benefit from the attentional boost that is triggered towards $\mathrm{T} 1$, and thus no Lag-1 sparing and no order errors are expected. However, Lunau and Olivers (2010) suggested that the attentional boost may be moved to another location (by means of a moving cue). Either way, this theoretical account cannot explain the complex pattern obtained in Exp. 1. In lag 1, left T2s showed a lower recognition rate compared to Central T2s, showing a location switch cost due to requirement to shift the attentional boost (Lunau \& Olivers, 2010). This lower T2 recognition was accompanied by high order error rate, suggesting that on occasions that left T2 was recognized, it benefited from a boost of attention that was strong enough to allow it a prior entry. Paradoxically, right T2s were not only better recognized (compared to left $\mathrm{T} 2 \mathrm{~s}$ ) but also better differentiated temporally, suggesting that the boost of attention T2 benefited from was enough to cause it to be better recognized, but not enough to cause a prior entry to consciousness. This pattern of RVF advantage cannot be reconciled with the model as is.

Another theoretical framework suggest that the $\mathrm{AB}$ is related to the parsing of visual input into attentional episodes (e.g., Bowman \& Wyble, 2007; Sperling \& Weichselgartner, 1995; Wyble, Bowman, \& Nieuwenstein, 2009; Wyble, Potter, Bowman, \& Nieuwenstein, 2011). By this theorizing, 
$\mathrm{AB}$ is regarded as the failure to create two episodes within a short interval, whereas Lag-1 sparing reflects the successful integration of the two targets in a single episode. Integration into one episode comes at a cost of losing information regarding T1-T2 temporal order (e.g., Akyürek et al., 2008, 2012; Bowman \& Wyble, 2007; Hommel \& Akyürek, 2005; Hommel et al., 2006; Visser et al., 1999). Also by this account, $\mathrm{T} 1$ and T2 must share the same spatial location in order to enter the same attentional episode, so Lag-1 sparing and T1T2 order errors could appear. This theoretical account allows for two different cases of $\mathrm{T} 2$ recognition; recognition of $\mathrm{T} 2$ could result from the entry of $\mathrm{T} 1$ and $\mathrm{T} 2$ into the same attentional episode, being accompanied by order errors, or from the opening of a new attentional episode for $\mathrm{T} 2$, resulting in a low order error rate. Finding order errors for left T2s suggest recognition of left T2s mainly relied on T2 entering the same attentional episode as T1. In contrast, low order error rate for right $\mathrm{T} 2 \mathrm{~s}$ suggest that they were more likely to reside within a new attentional episode. This implies a spatio-temporal asymmetry, with left targets being more likely to be perceived in the same location as central targets, while right targets being more likely to be perceived as spatially distinct.

The results of Exp. 3 and Exp. 4 pose a problem for our interpretation of Exp. 1 results in terms of the attentional episodes framework. While results revealed an RVF advantage, order error rate decreased for left $\mathrm{T} 2 \mathrm{~s}$ to a similar rate as on the right. In contrast to Exp. 1, in Exps. 3 and $4 \mathrm{~T} 1$ was masked by a distractor. This distractor could serve as a spatial mark differentiating left from center. Thus, we suggest that for left T2s, T1-mask allowed participants to infer temporal order indirectly based on the spatial location of the perceived target (i.e., if it is next to a distractor, it is on the side, therefore it must be T2).

There is an apparent contradiction between our RVF advantage and a corpus of studies that reported an LVF advantage in dual-stream RSVP (reviewed in Verleger \& Smigasiewicz, 2015). We will consider three possible explanations to this contradiction. First, one might dismiss this discrepancy attributing it to the differences in reading habits between our right-to-left reading participants (Hebrew readers) to the left-to-right reading participants in the previous studies. However, the disappearance of RVF advantage in Exp. 2 suggests this is not the case. Importantly, support for the suggestion that the LVF advantage does not relate to reading direction habits was received by a study that showed similar levels of LVF advantage for readers of a left-to-right language (German readers), a right-to-left language (Hebrew readers) and a top-to-bottom language (Taiwanese Chinese readers) (Smigasiewicz et al., 2010).

Another possibility is that the contradiction between RVF and LVF advantages is related to a methodological difference between the current study and previous experiments. While in the studies of Verleger and colleagues, T1 was presented in one of two side streams, in our experiments T1 was always presented in a central stream. Following a suggestion that central events on RSVP are handled mainly by the right hemisphere (RH; e.g., Verleger et al., 2013), in our study, central T1 is presumably processed by the RH. Then, left T2 would require the RH to deal with two targets. These high demands on the RH (e.g., Scalf et al., 2007) would result in left T2s being more often blinked, and when recognized, being more susceptible to order errors. One might even speculate that being processed by the same hemisphere they are more prone to enter the same attentional episode. In contrast, right $\mathrm{T} 2 \mathrm{~s}$ would be handled by the left hemisphere (LH), and dividing attentional demands between the two hemispheres should result in better T2 recognition (e.g., Scalf et al., 2007) and fewer order errors, as the two targets are more likely to create two separate attentional episodes. Nevertheless, this does not explain why we got no RVF advantage in Exp. 2 although T1 was presented in the central stream also in this experiment. Therefore, because no RVF advantage was found in Exp. 2, the asymmetry that was found in Exps. 1, 3 and 4 does not seem to be related to the central presentation of $\mathrm{T} 1$ per se.

We lean towards a third account, highlighting the role of bottom-up attention. As previous studies demonstrated that LVF advantage disappeared when the relative salience of T2 was increased (Scalf et al., 2007; Smigasiewicz et al., 2014; Verleger \& Smigasiewicz, 2015), the current study sought for visual field asymmetry in the $\mathrm{AB}$ under conditions of the bottom-up saliency of T2. Indeed, an RVF advantage emerged when $\mathrm{T} 2$ was highly salient, by means of abrupt onset (Exps. 1, 3 and 4), and was eliminated once T2 was embedded within a stream of similar distractors (Exp. 2), which immensely reduced its saliency. We suggest that the inconsistencies between RVF and LVF advantages might reflect differences in spatiotemporal dynamics under conditions of bottom-up vs. top-down attention. While bottom-up attention seems to be biased towards the RVF, top-down attention seems to be biased towards the LVF. Note however, that, unlike the RVF advantage found with dual-stream RSVP in Verleger and colleagues' studies, an RVF advantage did not emerge in our Exp. 2 , where targets were embedded in three full concurrent streams. This implies that top-down attentional selection requirements are not enough for RVF advantage to emerge. Future studies should further clarify under what condition an RVF advantage occur in the AB.

The distinction between bottom-up and top-down attentional gating or automatic and controlled attentional gating was previously suggested, asserting that bottom-up salient stimulus can directly signal attention to initiate a new attentional gate (see Shih, 2000, 2008; Shih \& Reeves, 2007, Weichselgartner \& Sperling, 1987). However, this account does not explain why targets presented in the RVF would benefit from bottom-up saliency more than targets presented in the LVF. Further studies should be conducted to elucidate the mechanisms behind RVF advantage in the $\mathrm{AB}$, and to test whether it characterizes bottom-up attention in general. 
Acknowledgements We wish to thank Sebastien Marti for useful comments on a previous version of this manuscript, and the anonymous reviewers for their enlightening comments that enormously improved this manuscript.

\section{References}

Akyürek, E. G., Eshuis, S. A., Nieuwenstein, M. R., Saija, J. D., Başkent, D., \& Hommel, B. (2012). Temporal target integration underlies performance at lag 1 in the attentional blink. Journal of Experimental Psychology: Human Perception and Performance, 38(6), 1448-1464.

Akyürek, E. G., Toffanin, P., \& Hommel, B. (2008). Adaptive control of event integration. Journal of Experimental Psychology: Human Perception and Performance, 34(3), 569-577.

Asanowicz, D., Śmigasiewicz, K., \& Verleger, R. (2013). Differences between visual hemifields in identifying rapidly presented target stimuli: Letters and digits, faces, and shapes. Frontiers in Psychology, 4, 1-13.

Bowman, H., \& Wyble, B. (2007). The simultaneous type, serial token model of temporal attention and working memory. Psychological Review, 114(1), 38-70.

Broadbent, D. E., \& Broadbent, M. H. (1987). From detection to identification: Response to multiple targets in rapid serial visual presentation. Perception \& Psychophysics, 42(2), 105-113.

Chun, M. M., \& Potter, M. C. (1995). A two-stage model for multiple target detection in rapid serial visual presentation. Journal of Experimental Psychology: Human Perception and Performance, 2l(1), 109-127.

Dux, P. E., \& Marois, R. (2009). The attentional blink: A review of data and theory. Attention, Perception, \& Psychophysics, 71(8), 16831700 .

Giesbrecht, B., \& Kingstone, A. (2004). Right hemisphere involvement in the attentional blink: Evidence from a split-brain patient. Brain and Cognition, 55(2), 303-306.

Hilkenmeier, F., Olivers, C. N., \& Scharlau, I. (2012). Prior entry and temporal attention: Cueing affects order errors in RSVP. Journal of Experimental Psychology: Human Perception and Performance, 38(1), 180-190.

Holländer, A., Corballis, M. C., \& Hamm, J. P. (2005). Visual-field asymmetry in dual-stream RSVP. Neuropsychologia, 43(1), 35-40.

Hollander, A., Hausmann, M., Hamm, J. P., \& Corballis, M. C. (2005). Sex hormonal modulation of hemispheric asymmetries in the attentional blink. Journal of the International Neuropsychological Society, 11(3), 263-272.

Hommel, B., \& Akyürek, E. G. (2005). Lag-1 sparing in the attentional blink: Benefits and costs of integrating two events into a single episode. The Quarterly Journal of Experimental Psychology Section A, 58(8), 1415-1433.

Hommel, B., Kessler, K., Schmitz, F., Gross, J., Akyürek, E., Shapiro, K., \& Schnitzler, A. (2006). How the brain blinks: Towards a neurocognitive model of the attentional blink. Psychological Research, 70(6), 425-435.

Jefferies, L. N., \& Di Lollo, V. (2009). Linear changes in the spatial extent of the focus of attention across time. Journal of Experimental Psychology: Human Perception and Performance, 35(4), 1020 1031.

Jefferies, L. N., Enns, J. T., \& Di Lollo, V. (2014). The flexible focus: Whether spatial attention is unitary or divided depends on observer goals. Journal of Experimental Psychology: Human Perception and Performance, 40(2), 465-470.

Jefferies, L. N., Ghorashi, S., Kawahara, J. I., \& Di Lollo, V. (2007). Ignorance is bliss: The role of observer expectation in dynamic spatial tuning of the attentional focus. Perception \& Psychophysics, 69(7), 1162-1174.

Jolicœur, P., \& Dell'Acqua, R. (1998). The demonstration of short-term consolidation. Cognitive Psychology, 36(2), 138-202.

Lunau, R., \& Olivers, C. N. (2010). The attentional blink and lag 1 sparing are nonspatial. Attention, Perception, \& Psychophysics, 72(2), 317-325.

Martens, S., \& Wyble, B. (2010). The attentional blink: Past, present, and future of a blind spot in perceptual awareness. Neuroscience \& Biobehavioral Reviews, 34(6), 947-957.

Olivers, C. N. L., Hilkenmeier, F., \& Scharlau, I. (2011). Prior entry explains order reversals in the attentional blink. Attention, Perception, \& Psychophysics, 73(1), 53-67.

Olivers, C. N. L., \& Meeter, M. (2008). A Boost and Bounce theory of temporal attention. Psychological Review, 115, 836-863.

Potter, M. C., Chun, M. M., Banks, B. S., \& Muckenhoupt, M. (1998). Two attentional deficits in serial target search: The visual attentional blink and an amodal task-switch deficit. Journal of Experimental Psychology: Learning, Memory, and Cognition, 24(4), 979-992.

Potter, M. C., Staub, A., \& O'Connor, D. H. (2002). The time course of competition for attention: Attention is initially labile. Journal of Experimental Psychology: Human Perception and Performance, 28(5), 1149-1162.

Raymond, J. E., Shapiro, K. L., \& Arnell, K. M. (1992). Temporary suppression of visual processing in an RSVP task: An attentional blink? Journal of Experimental Psychology: Human Perception and Performance, 18(3), 849-860.

Scalf, P. E., Banich, M. T., Kramer, A. F., Narechania, K., \& Simon, C. D. (2007). Double take: Parallel processing by the cerebral hemispheres reduces attentional blink. Journal of Experimental Psychology: Human Perception and Performance, 33(2), 298-329.

Shapiro, K. L., Raymond, J. E., \& Arnell, K. M. (1994). Attention to visual pattern information produces the attentional blink in rapid serial visual presentation. Journal of Experimental psychology: Human perception and performance, 20(2), 357-371.

Shih, S. I. (2000). Recall of two visual targets embedded in RSVP streams of distractors depends on their temporal and spatial relationship. Perception \& Psychophysics, 62(7), 1348-1355.

Shih, S. I. (2008). The attention cascade model and attentional blink. Cognitive Psychology, 56(3), 210-236.

Shih, S., \& Reeves, A. (2007). Attentional capture in rapid serial visual presentation. Spatial Vision, 20(4), 301-315.

Śmigasiewicz, K., Asanowicz, D., Westphal, N., \& Verleger, R. (2014). Bias for the left visual field in rapid serial visual presentation: Effects of additional salient cues suggest a critical role of attention. Journal of Cognitive Neuroscience, 27(2), 266-279.

Śmigasiewicz, K., Shalgi, S., Hsieh, S., Möller, F., Jaffe, S., Chang, C. C., \& Verleger, R. (2010). Left visual-field advantage in the dual-stream RSVP task and reading-direction: A study in three nations. Neuropsychologia, 48(10), 2852-2860.

Sperling, G., \& Weichselgartner, E. (1995). Episodic theory of the dynamics of spatial attention. Psychological Review, 102(3), 503-532.

Verleger, R., Dittmer, M., \& Śmigasiewicz, K. (2013). Cooperation or competition of the two hemispheres in processing characters presented at vertical midline. PloS One, 8(2), e57421.

Verleger, R., Möller, F., Kuniecki, M., Śmigasiewicz, K., Groppa, S., \& Siebner, H. R. (2010). The left visual-field advantage in rapid visual presentation is amplified rather than reduced by posterior-parietal rTMS. Experimental Brain Research, 203(2), 355-365.

Verleger, R., \& Śmigasiewicz, K. (2015). Consciousness wanted, attention found: Reasons for the advantage of the left visual field in identifying T2 among rapidly presented series. Consciousness and Cognition, 35, 260-273.

Verleger, R., Śmigasiewicz, K., \& Möller, F. (2011). Mechanisms underlying the left visual-field advantage in the dual stream RSVP task: 
Evidence from N2pc, P3, and distractor-evoked VEPs. Psychophysiology, 48(8), 1096-1106.

Verleger, R., Sprenger, A., Gebauer, S., Fritzmannova, M., Friedrich, M., Kraft, S., \& Jaśkowski, P. (2009). On why left events are the right ones: Neural mechanisms underlying the left-hemifield advantage in rapid serial visual presentation. Journal of Cognitive Neuroscience, 21(3), 474-488.

Visser, T. A., Bischof, W. F., \& Di Lollo, V. (1999). Attentional switching in spatial and nonspatial domains: Evidence from the attentional blink. Psychological Bulletin, 125(4), 458-469.

Visser, T. A., Zuvic, S. M., Bischof, W. F., \& Di Lollo, V. (1999). The attentional blink with targets in different spatial locations. Psychonomic Bulletin \& Review, 6(3), 432-436.

Weichselgartner, E., \& Sperling, G. (1987). Dynamics of automatic and controlled visual attention. Science, 238, 778-780.
Wyble, B., Bowman, H., \& Nieuwenstein, M. (2009). The attentional blink provides episodic distinctiveness: Sparing at a cost. Journal of Experimental Psychology: Human Perception and Performance, 35(3), 787-807.

Wyble, B., Potter, M. C., Bowman, H., \& Nieuwenstein, M. (2011). Attentional episodes in visual perception. Journal of Experimental Psychology: General, 140(3), 488-505.

Yantis, S. (1993). Stimulus-driven attentional capture. Current Directions in Psychological Science, 2, 156-161.

Yantis, S., \& Johnson, D. N. (1990). Mechanisms of attentional priority. Journal of Experimental Psychology: Human Perception and Performance, 16(4), 812-825.

Yantis, S., \& Jones, E. (1991). Mechanisms of attentional selection: Temporally modulated priority tags. Perception \& Psychophysics, 50(2), 166-178. 